\title{
Structure of SDS micelles with propylene carbonate as cosolvent: a PGSE-NMR and SAXS study
}

Giuseppe Colafemmina, Daniela Fiorentino, Andrea Ceglie, Emiliano Carretti, EmilianoFratini, Luigi Dei, Piero Baglioni, Gerardo Palazzo

\section{Supplementary information}

Representative conductivity and ion-selective electrode (ISE) measurements.

The influence of propylene carbonate on the self-assembly of SDS was investigated by means of conductivity ( $\kappa$, specific conductance) measurements. In Fig. SI-1 is shown the $\kappa$ vs. $c$ plot (c being

Figure SI-1

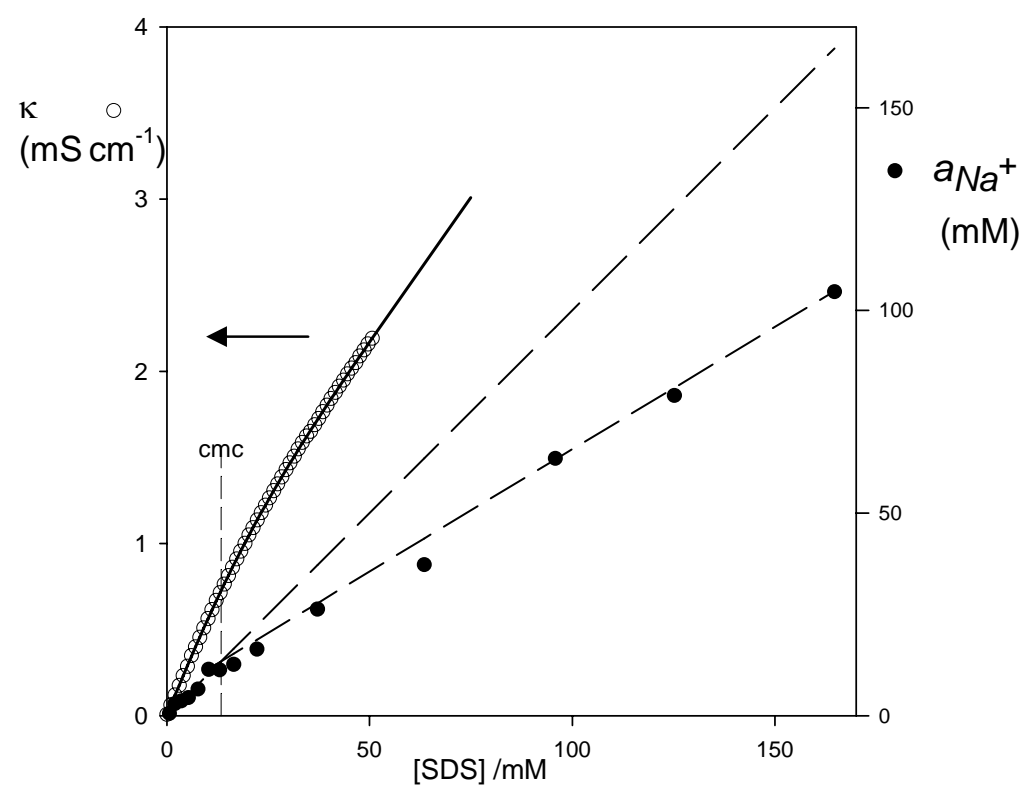

\section{Figure SI-1}

- Specific conductivity ( $\kappa$, left ordinate) vs. c for SDS dissolved $\mathrm{PC} /$ water solution ( $\mathrm{PC}=14 \% \mathrm{wt}$ ). Continuous curves denote the fit of experimental data to the function 1 of the main text: The best fit parameters are: $\Lambda_{\mathrm{S}}=68 \mathrm{~S} \mathrm{~cm}^{2} \mathrm{M}^{-1} ; \Lambda_{\text {mic }}=42 \mathrm{~S} \mathrm{~cm}^{2} \mathrm{M}^{-1} ; \sigma=0.68 \mathrm{mM} ; \mathrm{cmc}=13 \mathrm{mM}$. For PC/water the best fit parameters are: $\mathrm{A}_{1}=6.1 \times 10^{-2} \mu \mathrm{S} \mathrm{cm}^{-1} \mathrm{mM}^{-1} ; \mathrm{A}_{2}=5.3 \times 10^{-2} \mu \mathrm{S} \mathrm{cm}^{-1} \mathrm{mM}^{-1} ; \sigma=4 \mathrm{mM} ; \mathrm{cmc}=13 \mathrm{mM}$. The location of cmc values is highlighted by vertical dashed line.

- Sodium ion activity measured by the ISE ( $a_{N a+}$, right ordinate), as a function of c. Below cmc $a_{N a+}$ is identical to the total surfactant concentration $c$, and the degree of micelle ionization $\alpha$ was 1 . Above the $\mathrm{cmc}$ $a_{N a+}=(1-\alpha) \mathrm{cmc}+\alpha c$ (see reference 7 of the main text) with $\alpha=0.604$;

the SDS concentration) representative of the SDS behavior in PC/water mixture $\left(\mathrm{g}_{\mathrm{PC}} /\left(\mathrm{g}_{\mathrm{PC}}+\mathrm{g}_{\mathrm{water}}\right)=\right.$ 14\%). Note that conductivity curve exhibits a weak curvature, This behavior, often found for surfactant with small aggregation number, make difficult a safe determination of cmc. For this reason the cmc-values have been evaluated by fitting the whole data set to equation 1 of the main 
text (thick continuous line). The sodium activity (right ordinate) measured by ISE allows the determination of the degree of micelle ionization.

Conductivity along the dilution path.

The self-diffusion data of Figure 4 (main text) suggest that extrapolation to high dilution (just above the $\mathrm{cmc}$ ) of the dilution path determined by PGSE-NMR experiments should correspond to a system prepared by dissolving SDS in the PC/water dilution mixture (this is because at the cmc the fraction of PC adsorbed by the micelles is negligible). This point can be tested by means of conductometry. In Figure SI-2 are compared the conductivity values measured upon dilution (open symbols; $10 \mathrm{mM}<[\mathrm{SDS}]<337 \mathrm{mM}$ ) with the values taken in the cmc determination, i.e. loading with SDS (from 0 to $40 \mathrm{mM}$; closed symbols) a solution PC/water with fixed composition ( $\mathrm{PC}=8 \mathrm{wt} \%$ ). The curve in Figure SI-2A is the extrapolation of the low SDS concentration data to equation 1 of the main text; it well describes also the conductivities at high concentration along the dilution path. Further insight can be gained by comparing the derivatives $\mathrm{d} \kappa / \mathrm{d} c$ of the data collected in the different experiments (Figure SI-2B). At low (but still finite) concentration the equivalent conductivity of an electrolyte follows the Kohlrausch's law, i.e. $\Lambda=\Lambda^{\circ}-\mathrm{A} \sqrt{ } c$ (where the constant $\mathrm{A}$ is independent on the concentration $c$ ). In terms of specific conductivity one has $\kappa=\Lambda^{\circ} \mathrm{C}-\mathrm{A} c^{3 / 2}$ and further differentiation with $c$ leads to

$$
\frac{d \kappa}{d c}=\Lambda^{\circ}-\frac{3}{2} A \sqrt{c}
$$

according to the equation above, we expect that above the $\mathrm{cmc} d \kappa / \mathrm{d} c$ should follow a straight line with intercept equals to the molar conductivity at infinite dilution of the micelles $\Lambda^{\circ}$ mic. Of course below the cmc the extrapolation of $\mathrm{d} \kappa / \mathrm{d} c$ at $c=0$ gives the limiting equivalent conductivity of SDS. Two point of Figure SI-2B deserve some comments. 1) extrapolation of the linear part of dilution data gives an intercept $\left(\Lambda^{\circ}\right.$ mic $)$ that is very close to the $\Lambda_{\text {mic }}$ found by fitting the data around the cmc to equation 1 of the main text. 2) All the data follow the same trend. This means that, although upon dilution we change continuously the composition of the aqueous mixture, the system behaves as expected for identical micelles diluted by a continuous phase at constant composition. 

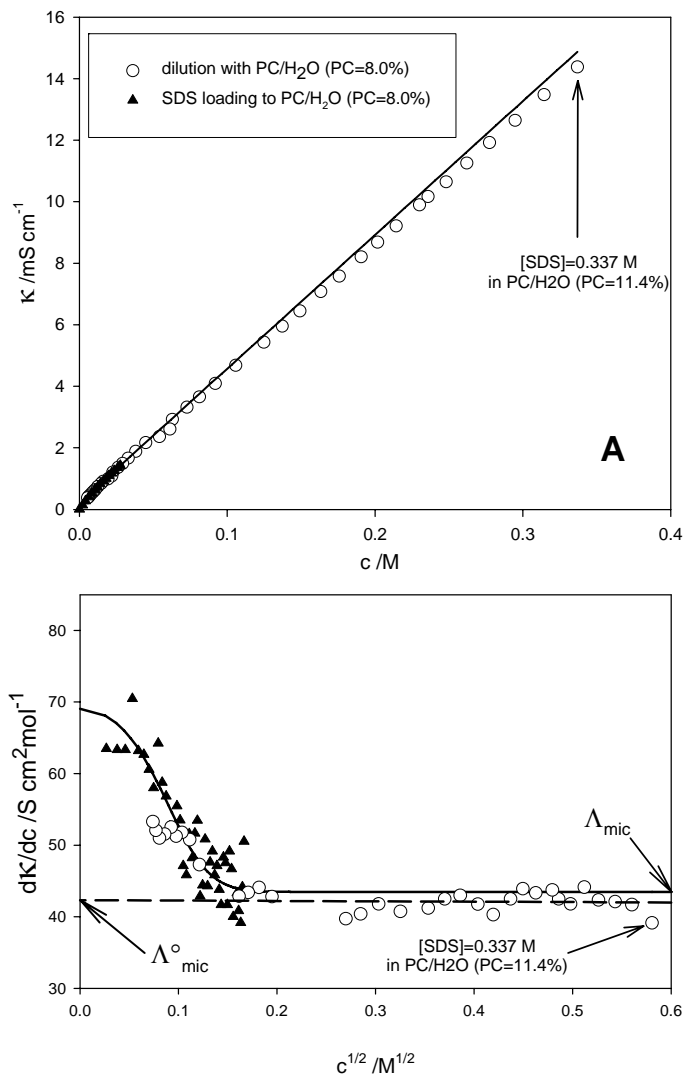

Figure SI-2 Conductivity along the same dilution path of Fig. 5 of the main text (open symbols) and upon SDS loading of a $\mathrm{PC} /$ water mixture at $\mathrm{PC}=8.0 \%$ (closed symbols).

A) conductivity vs. SDS concentration; the curve is the best fit (equation 1) of the data collected upon SDS loading.

B) $\mathrm{d} \kappa / \mathrm{dc}$ vs. the square root of surfactant concentration. The curve is the derivative of equation 2 ; the value of the parameter $\Lambda_{\mathrm{mic}}$ is shown on the right. The dashed straight line is the fit (c>40 mM) of data collected upon dilution according to equation 7 ; the intercept $\Lambda^{\circ}{ }_{\text {mic }}$ is shown on the left. 